\title{
Whether Platelet Count Really Influences the Outcome of Dengue Patients? A Study Done at Tertiary Care Centre in North Karnataka Region
}

\author{
Channamma Goudagaon ${ }^{1}$, Surajsingh Ravindra Taur ${ }^{2}$ \\ ${ }^{1}$ Department of General Medicine, M.R. Medical College, Kalaburagi, Karnataka, India. \\ ${ }^{2}$ Department of General Medicine, M.R. Medical College, Kalaburagi, Karnataka, India.
}

\section{ABSTRACT}

\section{BACKGROUND}

In many parts of India, Dengue has become a major public health problem and Kalaburagi District in North Karnataka region was previously not known to be an endemic area for dengue. Three clinical syndromes, classic dengue fever, dengue haemorrhagic fever and dengue shock syndrome are seen with dengue virus infection. This present study was undertaken to find out whether platelet count really influences the outcome of dengue patients in a tertiary health care centre in Kalaburagi, Karnataka.

\section{METHODS}

About hundred patients admitted in our hospital with positive Dengue NS1 and Dengue IgM were selected. The follow up of patients is done from the onset of fever to up to twelve days or until their recovery according to discharge criteria of WHO whichever of them is earlier.

\section{RESULTS}

In this study, 100 cases were studied, out of which Dengue fever was seen in 73 cases, Dengue Hemorrhagic Fever in 17 cases and Dengue Shock Syndrome in 10 cases was seen based on WHO criteria. In this study, 99 percent patients had fever. In this study 73 percent of patients were Dengue NS1 Positive and 27 percent of patients were Dengue IgM Positive. In present study, continuously increasing pattern of platelet count was seen in 55 percent cases; initial fall then rise of platelet count was seen in 38 percent cases and persistently low pattern of platelet count was seen in 7 percent cases. This study showed that 30 percent of patients had bleeding manifestations and bleeding manifestations were maximum in patients having low platelet counts. In the present study, $92 \%$ cases improved and $8 \%$ cases expired.

\section{CONCLUSIONS}

Among the three clinical syndromes, Dengue fever was more common than dengue haemorrhagic fever and dengue shock syndrome. It was found that platelet count influenced the outcome of dengue patients. Management of dengue case is mainly supportive.

\section{KEY WORDS}

Thrombocytopenia, Dengue Fever, Dengue Haemorrhagic Fever, Dengue Shock Syndrome, Dengue NS1, Dengue IgM, DHF, DSS, WHO
Corresponding Author:

Dr. Surajsingh Ravindra Taur, Room No. 113, PG Boys Hostel,

Basaweshwar Teaching and General Hospital, Sedam Road, Kalaburagi-585105, Karnataka, India.

E-mail:srtaur@gmail.com

DOI: $10.14260 / \mathrm{jemds} / 2020 / 0000$

Financial or Other Competing Interests: None.

How to Cite This Article:

Goudagaon C, Taur SR. Whether platelet count really influences the outcome of dengue patients? a study done at tertiary care centre in North Karnataka Region. J. Evolution Med. Dent. Sci. 2020;9(14): 1167-1170, DOI:
Submission 04-01-2020,

Peer Review 15-03-2020,

Acceptance 21-03-2020,

Published 06-04-2020. 


\section{BACKGROUND}

Dengue is a homonym for the African ki denga pepo, which appeared in English literature during an 1827-28 Caribbean outbreak. The word dengue came from denga or dyengo which in Africa means haemorrhage. Benjamin Rush in 1789 has published the first clinical report of Dengue. ${ }^{1}$ "The pains which accompanied this fever were exquisitely severe in the head, back, and limbs. The pains in the head were sometimes in the back parts of it, and at other times they occupied only the eyeballs. In some people, the pains were so acute in their backs and hips that they could not lie in bed. A few complained of their flesh being sore to the touch, in every part of the body. From these circumstances, the disease was sometimes believed to be rheumatism. But it's more general name among all classes of people was break-bone fever". Benjamin Rush (1745-1813).

Due to the presence of symptoms of myalgia and arthralgia the term break-bone fever is coined by him. ${ }^{2}$ Dengue fever is distributed world-wide, involving nearly all tropical and subtropical countries, and hence has many names like-dandy fever, Denguero, denga, dunga, break-bone fever, bouguet, seven day fever, bonon, chapenonada, Knieueble, Tokkive- ana, Mal de genoux, homa mguu, and coup-d-barre. ${ }^{3}$ Dengue virus (DENV) is single stranded RNA virus of genus Flavivirus and is arthropod borne. It is comprised of 4 antigenically distinct serotypes but are closely related, DENV-1, $-2,-3$, and -4 . Presently no specific therapies or vaccines are available to treat diseases or to prevent DENV transmission. Illnesses caused by DENV infection include undifferentiated fever, dengue fever (DF), dengue haemorrhagic fever (DHF), and dengue shock syndrome (DSS). ${ }^{4}$

Worldwide Dengue ranks as the most important mosquito-borne viral disease. According to current estimated reports, at least 112 countries are endemic for Dengue and in tropics and sub-tropics about $40 \%$ of the world populations (2.5-3 billion people) are at risk. Worldwide 100 million cases of dengue fever and about half a million cases of dengue haemorrhagic fever occur annually. Early recognition and prompt initiation of treatment are very necessary if morbidity and mortality related to disease are to be limited. 5

In Dengue fever (DF)/ Dengue Haemorrhagic Fever (DHF) and dengue shock syndrome (DSS) platelet count is a predictive as well as recovery parameter. ${ }^{6}$ Rain, temperature an relative humidity as the major and important climatic factors which alone or collectively be responsible for outbreak and the drastic fall in platelet count is life threatening in Dengue. ${ }^{7}$ Platelet count can be used to predict the complication and duration of hospital stay required and hence better use of resources is necessary. ${ }^{8}$ Thrombocytopenia the routine clinical parameter mainly was associated with severity of dengue fever. Early in the course of the illness to predict and manage dengue infection severity these parameters may be used to develop future scoring system. ${ }^{9}$

Dengue haemorrhagic fever can be distinguished from dengue fever (DF) by the presence of increased vascular permeability (plasma leakage syndrome) and marked thrombocytopenia $(<100,000 / \mu \mathrm{l})$ associated with bleeding, hepatomegaly and or abnormal liver function. Early diagnosis and immediately replacement of fluid should be done as the outcome of DHF and DSS largely depends on it.

We wanted to evaluate as to whether platelet count influences the outcome of dengue patients in Tertiary Health Centre at Kalaburagi in North Karnataka Region.

\section{METHODS}

This is a prospective longitudinal study conducted among patients admitted to a Multispeciality Hospital at Kalaburagi in North Karnataka Region who had been confirmed to be Dengue positive (IgM, NS1). About hundred patients admitted to the hospital with positive Dengue NS1 and Dengue IgM were selected using purposive sampling techniques during the study period. The follow up of patients is done from the onset of fever to up to twelve days or until their recovery according to discharge criteria of WHO whichever of them is earlier (national guidelines for management of dengue fever 2014). The study was approved by IEC and informed consent was obtained.

\section{Exclusion Criteria}

1. Patients with dengue with any other associated infections. E.g.: Malaria with positive dengue, Enteric fever with positive dengue

2. Patients with dengue with any other medical comorbidities. E.g.: Diabetes mellitus, hypertension, ITP

\section{Statistical Analysis}

In the present study Descriptive and inferential statistical analysis has been carried out. Analysis of the results was done by using SPSS version 18 (IBM Corporation, SPSS Inc., Chicago, IL, USA). Results are presented in Frequency (percentage) on categorical measurements. Chi-square test/Fischer-exact test was used for inferential statistics purpose. Significant P value was considered to be less than 0.05 .

\section{RESULTS}

Globally Dengue fever is a major public health problem. Epidemics of dengue fever are becoming more frequent in India. Classical dengue fever is an acute febrile illness is seen in maximum number of patients, but in a small percentage of cases a more severe form of disease known as Dengue haemorrhagic fever and dengue shock syndrome occurs. Early recognition and prompt management of this killer disease is very necessary to prevent case mortality. In our hospital, 100 patients admitted with Dengue NS1 Positive and Dengue IgM positive were studied.

In this study, 73 percent of patients came under Dengue fever, 17 percent patients under DHF and 10 patients had DSS and respective platelet count according to it. Above table shows that in this study, $\mathrm{PCV}<40$ was seen in 85 percent cases and PCV 40-45 was seen in 8 percent cases and PCV $>45$ was seen in 7 cases. 


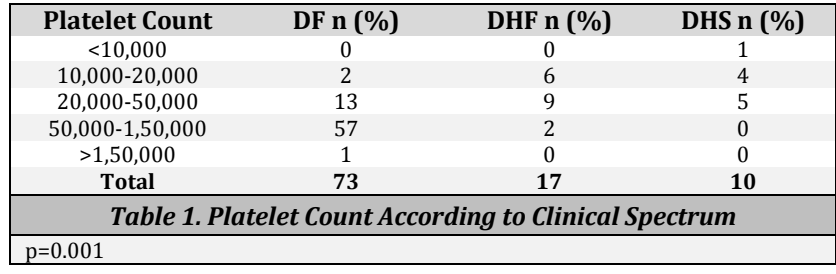

\begin{tabular}{|ccc|}
\hline PCV & Frequency & Percentage \\
$<40$ & 85 & 85 \\
$40-45$ & 8 & 8 \\
$>45$ & 7 & 7 \\
Total & $\mathbf{1 0 0}$ & $\mathbf{1 0 0}$ \\
\hline \multicolumn{3}{|}{} \\
\hline \multicolumn{3}{|c}{ Table 2. Haematocrit $($ PCV $)$} \\
\hline
\end{tabular}

\begin{tabular}{|ccc|}
\hline & Frequency & Percentage \\
Continuously Increasing & 55 & 55 \\
Initial fall then rise & 38 & 38 \\
Persistently low & 7 & 7 \\
Total & $\mathbf{1 0 0}$ & $\mathbf{1 0 0}$ \\
\hline \multicolumn{2}{|c|}{ Table 3. Pattern of Platelet Count } \\
\hline
\end{tabular}

Above Table shows that in present study, continuously increasing pattern of platelet count seen in 55 percent cases, Initial fall then rise of platelet count seen in 38 percent cases and persistently low pattern of platelet count seen in 7 percent cases It was observed that maximum cases were from the age group 21-30 year group 48 in number, as dengue is seen more in younger age group. 74 males and 26 females were included. Bleeding manifestation was seen in 30 percent of patients, pallor was seen in 15 percent patients and icterus was seen in 1 percent patients and oedema in 2 percent patients and 1 percent had both pallor and icterus and 1 percent had all 3

In this study 99 percent patients had fever, hypotension was seen in 12 percent patients, tachycardia was seen in 29 percent of patients. On RS examination 11 percent of patients had crepts and 1 percent patients had pleural effusion. On CVS examination S3 was heard in 2 percent of patients. On PA examination organomegaly and ascites was seen in 21 percent of patient. On CNS examination study Altered sensorium seen in 1 percent patients and Neck Rigidity seen in 3 percent of patients In present study, 73 percent of patients were diagnosed as Dengue fever, 17 percent patients diagnosed as DHF and 10 patients diagnosed as DSS. Hb $<15$ was seen in 15 percent of patients, $\mathrm{Hb} 10-12$ seen in 23 percent of patients, $\mathrm{Hb} 12.1-14$ seen in 32 percent of patients, $\mathrm{Hb} 14.1-16$ seen in 26 percent of patients, $\mathrm{Hb}>16$ was seen in 4 percent patients. Hb level less than 10 was seen in 11 cases of DF and 3 cases of DHF and 1 case of DSS. Hb level more than 16 was seen in 2 cases of DF and 2 cases of DHF

It was seen that most of the patients (54) had a total count of $4000-10,000$ cells $/ \mathrm{mm}^{3}$. In 10 patients leukocytosis was seen and in 36 patients leucopenia was seen. Creatinine levels were $<1.4$ in 96 percent patients and creatinine $>1.4$ in 6 percent patients Creatinine levels less than 1.4 was seen in 70 cases of DF and 14 cases of DHF and 9 cases of DSS, Creatinine levels more than 1.4 was seen in 2 cases of DF and 3 cases of DHF and 1 case of DSS. Bilirubin $<0.9$ was seen in 73 percent of patients and bilirubin 0.9-1.2 in 19 percent of patients and bilirubin $>2$ in 8 percent of patients. Bilirubin level less than 0.9 was seen in 55 of DF cases and 14 of DHF cases and 4 of DSS cases and Bilirubin level more than 2 was seen in 3 of DF cases and 3 of DSS cases. It was seen that $\mathrm{PT} / \mathrm{INR}$ was deranged in 3 percent of patients $\mathrm{PCV}<40$ was seen in 85 percent cases and PCV 40-45 was seen in 8 percent cases and PCV $>45$ was seen in 7 cases. 73 percent of patients were Dengue NS1 Positive and 27 percent of patients were Dengue IgM Positive. It was seen that 33 percent of patients had complications like AKI, Serositis, Polyserositis, encephalitis, hepatomegaly, splenomegaly, ascites, hepatitis, myositis and 99 percent of patients were having platelet count less than 1.5 lakhs and 1 percent patients were having platelet count more than 1.5 lakhs

It showed that 30 percent of patients had bleeding manifestations and bleeding manifestations were maximum in patients having low platelet counts. In present study 2 cases of DHF expired and 6 cases of DSS expired showing that maximum mortality was seen in cases with DSS. Continuously increasing pattern of platelet count seen in 55 percent cases, Initial fall then rise of platelet count seen in 38 percent cases and persistently low pattern of platelet count seen in 7 percent cases.

\section{DISCUSSION}

In the present study, Dengue fever was seen in $73 \%$ of the study population and Dengue haemorrhagic fever was seen in $17 \%$ and Dengue shock syndrome was seen in $10 \%$ of study population. In a study done by Neerja $\mathrm{M}$ et al the prevalence of DF, DHF, DSS was found to be $85 \%, 5 \%, 10 \%$ respectively. Pancharoen et al has done a study it was showing that the incidence of DHF was high i.e., 60.4\%. The results of this present study matches with that of a study done by Neerja $\mathrm{M}$ et al. From these observations of studies, we can come to a judgement that geographical area is an important factor according to which the incidence of each clinical spectrum varies. The area where we have conducted the study is not a known endemic area for dengue fever.

\begin{tabular}{|cccc|}
\hline No. & Author & Place & Platelet \\
1 & Cherian T et al $^{78}$ & Hyderabad & $94.7 \%$ \\
2 & Singh NP $79^{1}$ & Delhi & $61.39 \%$ \\
3 & Khan E et al $^{6}$ & Thailand & $81.4 \%$ \\
4 & Nandini et al 19 & Kolkata & $55.6 \%$ \\
5 & Present study & Gulbarga & $85 \%$ \\
\hline \multicolumn{3}{|c|}{ Table 4. Comparison of Thrombocytopenia $^{\text {in the Present Study with Other Studies }}$} \\
\hline
\end{tabular}

In present study, $85 \%$ patients had thrombocytopenia. Studies by Cherian $\mathrm{T}$ et al, Nandini et al, Khan E et al and Singh NP et al showed the incidence of thrombocytopenia in $94.7 \%, 55.6 \%, 81.4 \%$ and $61.39 \%$ respectively. The results of present study was correlated with the above mentioned studies. The association of dengue virus infection with thrombocytopenia has been found to be significant $(p<0.001)$

In our study, distribution of lowest platelet count during hospitalization, we found 01 patient had platelet count in range of $>1,50,000$ followed by 27 patients in range of 20,001-50000, 59 patients had platelet count between $51000-1,50,000$ there were 12 patients in the range of $10,001-20,001$ and 01 cases below 10,000 platelet count. This range correlated with bleeding tendencies and manifestations.

In present study features of shock were seen in $10 \%$ patients. A study conducted by Nimmanitya et al ${ }^{7}$ showed that $35 \%$ patients had incidence of shock and the study done by Nandini et al showed that $11.5 \%$ patients had incidence of shock. 


\begin{tabular}{|cccc|}
\hline SI. No. & Study & Place & Shock \\
1 & Nimmanitya et al & SEAR & $35 \%$ \\
2 & Nandini et al & Kolkata & 11.5 \\
3 & Present study & Gulbarga & $10 \%$ \\
\hline
\end{tabular}

Table 5. Comparison of Shock in the Present Study with Other Studies

\section{Haematocrit}

In the present study, the haematocrit ranged from $32-60 \%$. The mean haematocrit value of dengue positive cases in my study was $40.7 \%$. In DHF and DSS, the haematocrit levels were increased accordingly. This correlated as per the WHO guidelines

\section{Features of Fluid Leakage}

In this study out of 100 patients, $1 \%$ patients were having pleural effusion, $2 \%$ patients were having pedal oedema, $10 \%$ patients were having ascites. These above mentioned results were compared and correlated with the studies done by Dash P K et al and Neerja et al. The supporting evidence of plasma leakage are pleural effusion, pedal oedema and ascites, the distinguishing feature of DHF and DSS as per WHO guidelines.

\section{Mortality Rate}

In present study $92 \%$ cases Improved and 8\% cases Expired. The study done by Nandini et al in Kolkata showed a case fatality rate came out to be $3.8 \%$., whereas in my study the fatality was 7 in number. All these patients had presented very late to the hospital and hence the delay in management. Most common presentation in the present study was dengue fever which was followed by other complicated forms such as dengue haemorrhagic fever and dengue shock syndrome. Most common presenting complaints were Fever, Headache, Retro-orbital pain, Arthralgia, Myalgia, rashes, low backache, However, few patients also presented with atypical presentation such as sore throat, cough, diarrhoea, vomiting, and. Breathlessness. Jaundice, bleeding was seen in patients with secondary dengue infection.

On examination, icterus, haemorrhagic spot, positive tourniquet test, hypotension, pleural effusion, ascites, are the common findings mainly associated with the complicated forms of dengue. In severe forms of dengue fever, bleeding, hepatitis, pneumonia, polyserositis, shock are the common complications seen. On investigations altered renal function test, liver function test, secondary dengue and on ultrasound abdomen gall bladder wall thickened and hepatosplenomegaly seen, and on chest radiogram, pleural effusion and pneumonia seen are associated with dengue haemorrhagic fever and dengue shock syndrome. It was found that platelet count alone does not correlate with severity of disease. Higher rate of complications had an association with secondary dengue infection. Patient should be closely watched for bleeding tendencies and features of plasma leakage such as pedal oedema, pleural effusion, ascites and should be aggressively and appropriately managed.

Hess test if comes positive should prompt close observation and early hospital referral, but if test is negative it does not exclude dengue infection. The treatment of dengue mainly focuses on appropriate fluid management and supportive care and monitoring of haemodynamic status and it plays a major role in outcome of the disease. The idea about advent, intensity, seasonal incidence, transmission season, warning signs and impending epidemic of dengue and DHF was obtained from Dengue Surveillance studies. A more correct information would be provided by active longitudinal serosurvey over large scale along with the study of vector competence and vector capacity. Early recognition and management will decrease the case fatality rate. Finally it was found that platelet count influenced the outcome of dengue patients.

\section{CONCLUSIONS}

Among the three clinical syndromes, Dengue fever was more common than dengue haemorrhagic fever and dengue shock syndrome. It was found that platelet count influenced the outcome of dengue patients. The management of dengue case is mainly supportive.

\section{ACKNOWLEDGEMENT}

Authors thank Dr. Basawaraj Mangshetty, HOD. The authors thank Dr. Amit and Dr. Uday for helping with statistical analysis. The authors also thank their colleagues in the Department of Medicine, the paramedical staff, co post graduate students from all departments, for their help during the study.

\section{REFERENCES}

[1] Perez JGR, Clark GG, Gubler DJ, et al. Dengue and dengue haemorrhagic fever. Lancet 1998;352(9132):971-7.

[2] Dengue. Guidelines for diagnosis, treatment, prevention and control. $2^{\text {nd }}$ edn, Geneva: World Health Organization, 2009: p. 1-144.

[3] World Health Organization, Dengue haemorrhagic fever: diagnosis, treatment and control. Geneva: WHO, 1986.

[4] Sun P, Kochel TJ. The battle between infection and hostimmune responses of dengue virus and its implication in dengue disease pathogenesis. Scientific World Journal 2013;2013:843469.

[5] Malavige GN, Fernando S, Fernando DJ, et al. Dengue viral infections. Postgrad Med J 2004;80(948):588-601.

[6] Jayashree K, Manasa GC, Pallavi P, et al. Evaluation of platelets as predictive parameters in Dengue fever. Indian J Hematol Blood Transfus 2011;27(3):127-30.

[7] Pruthvi D, Shashikala P, Shenoy V. Evaluation of platelet count in dengue fever along with seasonal variation of dengue infection. J Blood Disorders \& Transfusion 2012;3(4):128.

[8] Figures compiled from various reports in WHO Regional Offices (AMRO, SEARO and WPRO).

[9] Sharma SK, Gupta BS, Devpura G, et al. Pulmonary haemorrhage syndrome associated with dengue haemorrhagic fever. A case report. J Assoc Physicians India 2007;55:729-30. 